\title{
Steady-state kinetics of the autocatalytic zymogen activation: A comparison with the Michaelis-Menten reaction mechanism
}

\author{
Malgorzata Anna Tyczynska ${ }^{1}$, Justin Eilertsen ${ }^{1}$, and Santiago Schnell ${ }^{1,2,3}$ \\ ${ }^{1}$ Department of Molecular $\&$ Integrative Physiology, University of Michigan Medical School, \\ Ann Arbor, Michigan, USA \\ ${ }^{2}$ Department of Computational Medicine \& Bioinformatics, University of Michigan Medical \\ School, Ann Arbor, Michigan, USA \\ ${ }^{3}$ E-mail: schnells@umich.edu
}

\begin{abstract}
A zymogen is an inactive precursor of an enzyme, which needs to go through a chemical change to become an active enzyme. The general intermolecular mechanism for the autocatalytic activation of zymogens is governed by the single-enzyme, single-substrate catalyzed reaction following the MichaelisMenten mechanism of enzyme action, where the substrate is the zymogen and product is the same enzyme catalyzing the reaction. In this article we investigate the nonlinear chemical dynamics of the intermolecular autocatalytic zymogen activation reaction mechanism, and compare it to that of the Michaelis-Menten reaction mechanism. We show that the intermolecular autocatalytic zymogen activation exhibits significant changes in reaction dynamics relative to the Michaelis-Menten reaction mechanism. These changes include differences in the number of conservation laws, number and stability of equilibrium states, altered structure of the invariant set that influences the long-time rate of the reaction, and qualitative evolution of the reaction depending strictly on the choice of initial conditions. We find a rate law, homologous to the Michaelis-Menten equation, to estimate the kinetic parameters of the intermolecular autocatalytic zymogen activation reaction mechanism, and derive the conditions for the validity for this rate law. Finally, we derive analytical expressions to estimate the timescale for the completion of the zymogen activation, which could have a practical application to calculate the molar enthalpy $\Delta H_{a p p}$ of the autocatalytic zymogen reaction in calorimetry assays.
\end{abstract}




\section{Introduction}

Steady-state systems provide a simple means of measuring the kinetic parameters of an enzyme catalyzed reaction. Perhaps the most widely recognized steady-state kinetics rate law is the Michaelis-Menten (MM) equation:

$$
\frac{\mathrm{d} s}{\mathrm{~d} t} \simeq-\frac{v s}{K_{M}+s},
$$

where $s$ denotes substrate concentration over time, $v$ is the limiting rate and $K_{M}$ is the Michaelis constant $[1,2]$. Equation (1) is typically derived by applying a mathematical model reduction technique - known as the quasi-steady-state approximation (QSSA) - to the MM reaction mechanism [3]

$$
S+E \underset{k_{-1}}{\stackrel{k_{1}}{\longrightarrow}} C \stackrel{k_{2}}{\longrightarrow} E+P
$$

In reaction scheme (2), the enzyme, $E$, binds to the substrate, $S$, and forms an intermediate complex, $C$. The complex then dissociates into either an enzyme molecule and a product, $P$, or to the original reactants. The constants $k_{1}, k_{-1}$ and $k_{2}$ denote reaction rates.

The MM equation (1) can be used to estimate $v$ and $K_{M}$ as long as specific reaction conditions are met $[3,4]$. Since the 1950s, steady-state kinetics has been extensively used to estimate kinetic parameters through progress curve or initial rate experiments $[5,6]$. The popularity of the MM equation (1) has substantially grown since the beginning of the century due to the implementation of systems biology approaches to model biochemical processes [7]. Nevertheless, despite the undeniable significance of the steady-state kinetics, one question remains unanswered for enzyme catalyzed reactions: do the properties of MM steady-state kinetics prevail for autocatalytic enzyme catalyzed reactions?

Zymogens are enzyme precursors (proenzymes) that can be activated through non-catalytic [8] or catalytic reactions, and have numerous biochemical functions. For example, they play a critical role in protein digestion by converting pepsin to pepsinogen [8,9], and trypsin to trypsinogen [10, 11, 12]. Activation can occur in three ways: (i) The inactive enzyme can be activated by another enzyme that cleaves off a peptide unit; this is the mechanism we consider in this work. (ii) The configuration of the zymogen can be changed in order to reveal the activation site. (iii) The inactive substance is activated when a coenzyme binds to the zymogen. A simple autocatalytic reaction that utilizes mechanism (i) is the activation of trypsinogen by trypsin $[13,14]$, while the activation of pepsinogen to active pepsin at low $\mathrm{pH}$ [5] follows mechanism (ii).

Mechanism (i) is represented schematically by

$$
S+E \underset{k_{-1}}{\stackrel{k_{1}}{\rightleftharpoons}} C \stackrel{k_{2}}{\longrightarrow} 2 E+W
$$

where $S$ is a zymogen, $E$ is an active enzyme, $W$ is peptide, and $k_{1}, k_{2}$ and $k_{-1}$ are rate constants. We refer to (3) as the intermolecular autocatalytic zymogen activation (IAZA) reaction. In addition, Fuentes [15] proposed a mechanism for zymogen activation that consists of two reaction pathways. One pathway consists of an intramolecular non-catalytic step in which the zymogen molecule disassociates into an active enzyme and a peptide

$$
S \stackrel{k_{d}}{\longrightarrow} E+W,
$$

while the additional catalytic step in the second pathway follows the IAZA reaction mechanism (3). More complicated models of zymogen activation have been analyzed in the context of a coupled enzyme assay that consists of an indicator reaction [16]. The model analyzed in [16] can be used to describe the activation of protein $\mathrm{C}$ by thrombin. However, neither reaction studied in [16] is autocatalytic, and thus a proper nonlinear analysis of a basic autocatalytic process through which zymogens are activated is currently lacking. This paper serves as a preliminary first step towards understanding such autocatalytic enzyme reaction mechanisms.

The significance of the IAZA reaction has motivated enzymologists to derive rate equations that describe the progress curves for zymogen activation [13], the steady-state or rapid equilibrium kinetic of zymogen action in the presence of reversible inhibitors [17], competitive and noncompetitive inhibitors [18], as well 
as complex inter- and intramolecular zymogen activation $[15,19,20]$. Although rate equations are derived under the presupposition of steady-state kinetics or QSSA, in many of studies of zymogen activation the steady-state kinetics assumption (or QSSA) has not been properly motivated through the traditional method of scaling [21, 22], or through more modern methods that have recently been developed [23, 24, 25, 26].

In the sections that follow, we investigate the nonlinear dynamics of the IAZA reaction mechanism (3) by comparing its behavior with the well characterized dynamics of the MM reaction mechanism (2). This is accomplished in consecutive steps. First, the phase-plane geometry of the IAZA reaction mechanism is inspected and compared to the MM reaction mechanism. Then, the dynamics of both reaction mechanisms are numerically simulated and analyzed via scaling arguments. We derive a quasi-steady-state (QSS) rate law that can be utilized to estimate the relevant kinetic parameters of the IAZA reaction mechanism through initial rate experiments. We also derive the conditions for the validity of QSSA, and discuss its range of applicability in experiments. Finally, we estimate the timescale for the completion of the zymogen activation that is valid when the QSSA holds; this has practical implications for calorimetry enzyme assays experimental design and interpretation. The results reported in this work can be applied to other zymogen reaction mechanisms, and serve as a preliminary foundation for the analysis of the reversible reaction, as well as the two-pathway mechanism proposed by Fuentes [15].

\section{The intermolecular autocatalytic zymogen activation reaction mechanism}

In this section, we apply the law of mass action to (3), which admits a mathematical model that describes the temporal dynamics of the IAZA reaction. This model permits us to investigate, as well as compare and contrast, the nonlinear dynamics and phase-plane geometries of the IAZA reaction mechanism (3) with the MM reaction mechanism (2). For the specific analysis of MM reaction mechanism (2), we refer the reader to references $[27,28,29]$.

Let $s:=s(t), e:=e(t)$ and $c:=c(t)$ denote the concentrations of $S, E$, and $C$ respectively. Applying the law of mass action yields:

$$
\begin{aligned}
\frac{\mathrm{d} s}{\mathrm{~d} t} & =-k_{1} e s+k_{-1} c, \\
\frac{\mathrm{d} c}{\mathrm{~d} t} & =k_{1} e s-\left(k_{-1}+k_{2}\right) c, \\
\frac{\mathrm{d} e}{\mathrm{~d} t} & =-k_{1} e s+\left(k_{-1}+2 k_{2}\right) c, \\
\frac{\mathrm{d} w}{\mathrm{~d} t} & =k_{2} c .
\end{aligned}
$$

Note that equations (5a)-(5c) admit a conservation law:

$$
\frac{\mathrm{d} s}{\mathrm{~d} t}+\frac{\mathrm{d} e}{\mathrm{~d} t}+2 \frac{\mathrm{d} c}{\mathrm{~d} t}=0
$$

Typically, experimental initial conditions are given by

$$
s(0)=s_{0}, \quad e(0)=e_{0}, \quad c(0)=0,
$$

and we will assume (7) holds in the analysis that follows. As we shall discuss later, the typical behavior of the IAZA system (5a)-(5d) depends on the relation between $s_{0}$ and $e_{0}$; this is illustrated on Figure 1. 

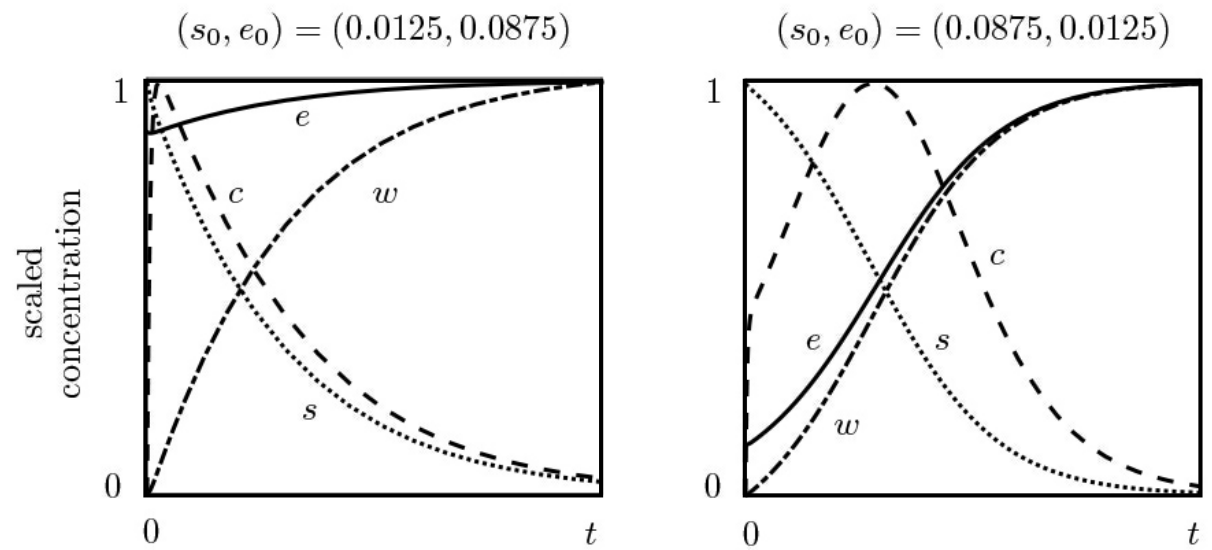

Figure 1: The relation between $s_{0}$ and $e_{0}$ influences the behavior of the IAZA time course trajectories (5a)-(5d). Each panel illustrates the $s, e, c$ and $w$ normalized with respect to their maximum value. Initial concentrations for $s$ and $e$ are given at the top of each figure panel and $w_{0}=c_{0}=0$ as assumed by (7). Other constants constants are $k_{1}=k_{2}=k_{-1}=1$. The initial conditions and parameter values are in arbitrary units for illustrative purposes.

Combining (6) with (7) yields $s+e+2 c=E_{T}$, where $E_{T}$ is the total concentration of zymogen and enzyme: $E_{T}=s_{0}+e_{0}$. Substituting $e=E_{T}-s-c$ into (5a)-(5b) yields

$$
\begin{aligned}
& \frac{\mathrm{d} s}{\mathrm{~d} t}=k_{1}\left[-\left(E_{T}-s\right) s+\left(K_{S}+2 s\right) c\right], \\
& \frac{\mathrm{d} c}{\mathrm{~d} t}=k_{1}\left[\left(E_{T}-s\right) s-\left(K_{M}+2 s\right) c\right],
\end{aligned}
$$

where $K_{S}=k_{-1} / k_{1}, K=k_{2} / k_{1}$ and $K_{M}=K_{S}+K$ denote the equilibrium, Van Slyke-Cullen and Michaelis constants of the IAZA reaction respectively. For simplicity, $W$ has been ignored since it is mathematically recoverable from $s$ and $c$. The constant $E_{T}$ is the unique conserved quantity that arises from (6)

$$
E_{T}=e+s+2 c .
$$

From the biochemical point of view, the initial conditions (7) define the constant $E_{T}$ as the sum of initial reactant concentrations $E_{T}=e_{0}+s_{0}$; this implies that $e=E_{T}$ upon completion of the reaction and imposes an upper bound on the initial concentration of zymogen: $0 \leq s_{0} \leq E_{T}$. Note that the presence of just one conservation law (9) in the IAZA reaction mechanism (3) is in contrast to the MM reaction (2), which has two independent conservation laws: one for the enzyme $\left(e+c=e_{0}\right)$, and another for the substrate $\left(s+c+p=s_{0}\right)$.

\subsection{The geometrical picture of intermolecular autocatalytic zymogen activation reaction mechanism}

The governing equations of the IAZA reaction mechanism (8a)-(8b) are nonlinear, and exhibit two equilibrium points:

$$
x_{1}^{*}=(0,0) \quad \text { and } \quad x_{2}^{*}=\left(E_{T}, 0\right) .
$$

The stability of equilibrium points (10) is conventionally determined by analyzing the Jacobian of the system (8a)-(8b) near these points. We find that $x_{1}^{*}$ is a stable point and $x_{2}^{*}$ is a saddle point. Since $x_{2}^{*}$ is a saddle point, it has one-dimensional stable $\mathcal{W}^{s}$ and unstable $\mathcal{W}^{u}$ manifolds. As we illustrate in Figure 2, the presence of $\mathcal{W}^{u}$ plays a critical role in uncovering the $c-s$ phase-plane geometry: $\mathcal{W}^{u}$ connects $x_{1}^{*}$ and $x_{2}^{*}$ and forms a heteroclinic orbit, $\Gamma_{\mathcal{W}}$, which is an invariant manifold that can potentially influence the 
long-time dynamics of the IAZA reaction. The existence of $\Gamma_{\mathcal{W}}$ is verified numerically using MATCONT open source software [30] (see [31] for details about the numerical algorithm utilized to track $\Gamma_{\mathcal{W}}$ ).

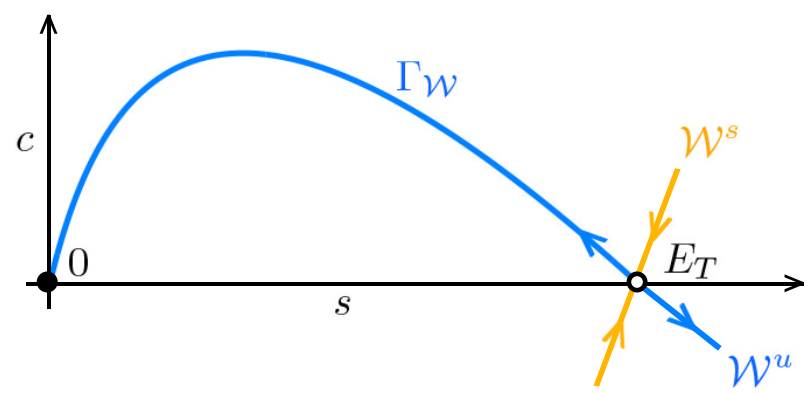

Figure 2: The geometry of the IAZA reaction mechanism (3) is characterized by the presence of heteroclinic orbit $\boldsymbol{\Gamma}_{\mathcal{W}}$. The system (8a)-(8b) has a stable equilibrium $x_{1}^{*}=(0,0)$ (the solid black circle) and the saddle equilibrium $x_{2}^{*}=\left(E_{T}, 0\right)$ (open circle). The heteroclinic orbit $\Gamma_{\mathcal{W}}$ that connects $x_{1}^{*}$ and $x_{2}^{*}$ is the solid blue curve, and the stable manifold corresponding to $x_{2}^{*}$ is the solid yellow curve.

Following [28], the shape of $\Gamma_{\mathcal{W}}$ in the $c-s$ phase-plane is interpreted relative to the $c$-nullcline, $\mathcal{N}_{c}(s)$, and the $s$-nullcline, $\mathcal{N}_{s}(s)$ :

$$
\mathcal{N}_{c}(s)=\frac{\left(E_{T}-s\right) s}{K_{M}+2 s}, \quad \mathcal{N}_{s}(s)=\frac{\left(E_{T}-s\right) s}{K_{M}-K+2 s} .
$$

The $c$-nullcline corresponds to the zeroth-order approximation to the QSS dynamics of (8a)-(8b), and the $s$-nullcline corresponds to the zeroth-order approximation to rapid equilibrium of (8a)-(8b). In the $c-s$ phase-plane, both nullclines, as well as $\Gamma_{\mathcal{W}}$, intersect at the equilibria (10). Due to the form of (11), the $c$ and $s$-nullclines are characterized by unimodal curves on the interval $\left[0, E_{T}\right]$, and with a maximum present at $s$ equal to $s_{c}^{*}$ and $s_{s}^{*}$ respectively. It is easy to verify that:

$$
s_{c}^{*} \simeq s_{s}^{*} \simeq \frac{E_{T}}{2} \text { when } K_{M} \gg E_{T} .
$$

The above condition for the equality of maxima can be written as

$$
\frac{E_{T}}{K_{M}} \ll 1,
$$

but we note that in fact $s_{c}^{*}$ is valid whenever $E_{T} \ll K_{S}$, which is less restrictive than (12). When (13) is satisfied, the nullclines assume their maximum values near $s=E_{T} / 2$, as shown in Figure 2. Furthermore, Figure 2 also reveals the nature of $\Gamma_{\mathcal{W}}$ : it remains under $\mathcal{N}_{c}$ for $s_{c}^{*}<s<E_{T}$, crosses it at $s \approx s_{c}^{*}$, and approaches the origin while staying between $\mathcal{N}_{c}$ and $\mathcal{N}_{s}$. The value of $s$ at which $\mathcal{N}_{s}$ and $\Gamma_{\mathcal{W}}$ intersect is only approximately $s_{c}^{*}$, since $\Gamma_{\mathcal{W}}$ has been tracked numerically using MATCONT [30]. Nevertheless, the precision of this proximity becomes less important if (13) holds, as $\Gamma_{\mathcal{W}}$ nearly aligns with $\mathcal{N}_{c}$. This observation is investigated further in the next section. 

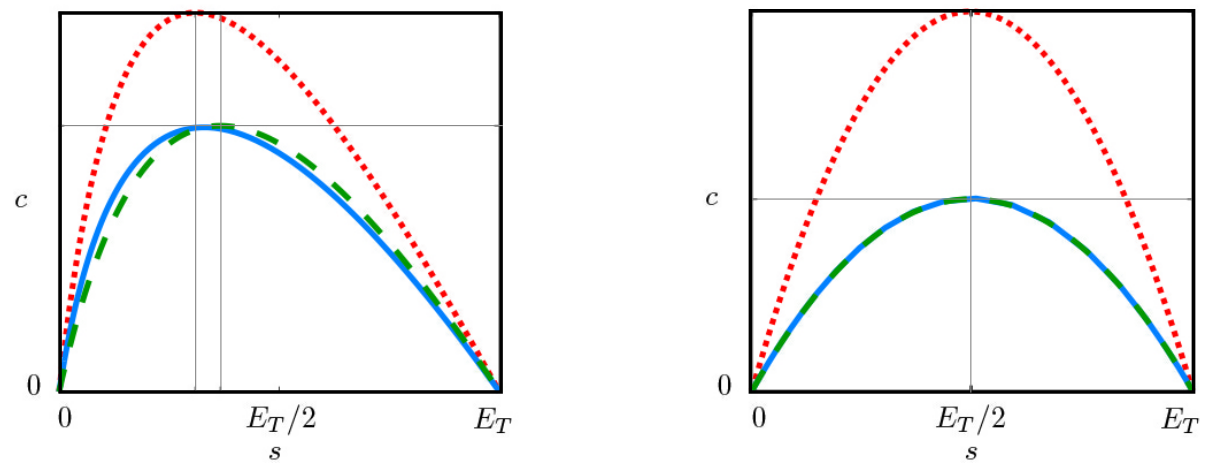

Figure 3: The effect of condition (13) to the $c-s$ phase plane geometry of the IAZA reaction mechanism (3). Each figure represents the rapid equilibrium nullcline $\mathcal{N}_{s}$ (dotted red line), quasi-steadystate nullcline $\mathcal{N}_{c}$ (stippled green line) and the heteroclinic orbit $\Gamma_{\mathcal{W}}$ (thick blue line). In both figures, the rate constants are $k_{1}=k_{-1}=k_{2}=1$, so $K_{M}=2$ with arbitrary units. On the figure to the left $E_{T} / K_{M}=1$, but to the right, $E_{T} / K_{M}=0.01$; thus, condition (13) is satisfied only in the latter case.

\subsection{Comparison of the autocatalytic zymogen activation with the Michaelis- Menten reaction}

Numerical evidence characterizes the dynamics of the MM reaction mechanism (2) by two sub-processes: an initial increase in $c$ followed by depletion of $s$ and $c$ [27]. The similarity in the geometry of MM and IAZA reaction mechanisms restricted to $0 \leq s<s_{c}^{*}$ suggests homologous dynamics of trajectories in this region, which is confirmed and illustrated in FIGURE 3 . This affinity vanishes when the IAZA dynamical trajectories are restricted to $s_{c}^{*} \leq s_{0} \leq E_{T}$, because the complex concentration changes over three sub-processes: an initial rise that coincides with the phase plane trajectory moving towards the $c$-nullcline, followed by another steady increase until the complex concentration reaches a maximum value and the phase plane trajectory intercepts the $c$-nullcline, after which, in the final step, the complex concentration steadily decreases. The second sub-process of the slow growth after the initial phase is unique to the IAZA reaction mechanism, as it is never present for the MM reaction mechanism. From an experimental point of view, this feature of the IAZA reaction mechanism illustrates that different choices in the initial concentrations of the chemical species can yield distinct differences in the progress curves of the reaction. 

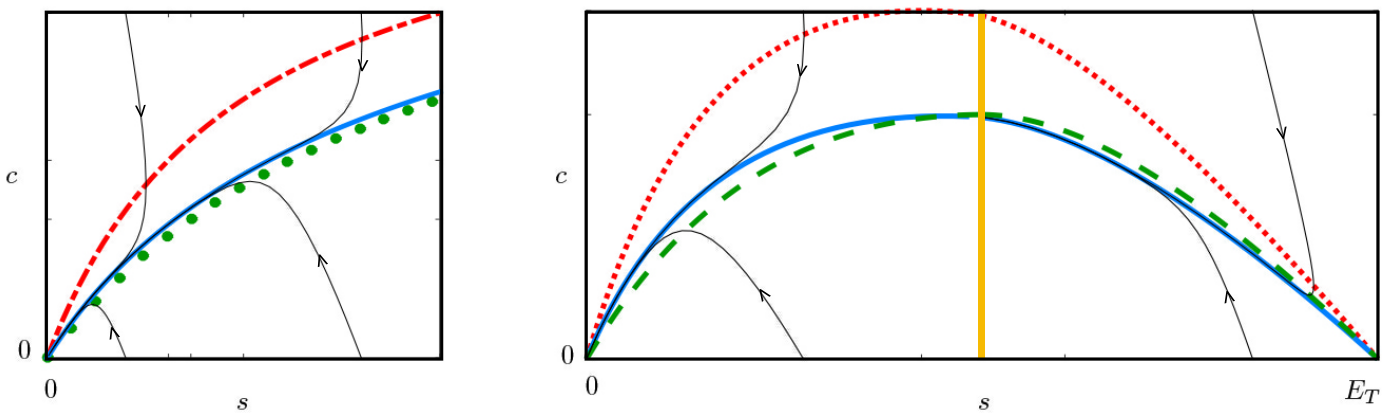

Figure 4: Comparison of the $c-s$ phase plane geometry of the MM reaction mechanism (2) and IAZA reaction mechanism (3). LEFT: The phase plane geometry of MM reaction mechanism (2), where the $\mathcal{N}_{s}, \mathcal{N}_{c}$ and slow invariant manifold are marked by a stippled red line, dotted green line and solid blue line, respectively. Right: The $c-s$ phase plane geometry of IAZA reaction mechanism (3) and the graphs follow the description as on Figure 3. On both panels, the thin black lines represent simulated trajectories with $\left(s_{0}, e_{0}\right)=(0.4,1.6)$ and $\left(s_{0}, e_{0}\right)=\left(1.6, s_{0}\right)$, thus $s_{0}+e_{0}=2$ for the IAZA reaction mechanism $\left(E_{T}=2\right)$. The numerical values are in arbitrary units for illustrative purposes. The solid orange line on the graph to the right divides the $c-s$ phase plane of the IAZA reaction mechanism into two parts: $0 \leq s<s_{c}^{*}$, where the dynamics is similar to MM reaction mechanism and to the $s_{c}^{*} \leq s<E_{T}$ with a dynamics unique to the IAZA reaction mechanism. The parameter values are $k_{1}=k_{2}=k_{-1}=1$ with arbitrary units, and $K_{M}=2=E_{T}$ in both panels. The slow manifold characterizing the geometry of MM reaction mechanism has been approximated by the method described in [28, 32].

\section{Application of the quasi-steady-state approximation to autocat- alytic zymogen activation}

Different model reduction techniques can be applied to derive expressions to model and monitor the MM reaction mechanism [33]. The most widely used model reduction technique in enzyme kinetics is the QSSA. The effectiveness of this technique requires meeting certain restrictions for the initial conditions and rate constants of the reaction $[27,34]$. In enzyme assays, the reaction achieves QSS when $c$ remains approximately constant over time, and the reaction rate changes relatively slowly. Rates are measured for a short period after reaching QSS by monitoring either the depletion of the substrate or the accumulation of product with time. Because the measurements are carried out for a very short period, the approximation $s \approx s_{0}$ can be made if there is an excess of substrate with respect to the enzyme in the reaction. From the mathematical point of view, under the QSSA, $s$ is the only variable that changes significantly over time, as $c$ remains in the QSS $[32,24,26]$.

In the previous section, we found substantial differences in the nonlinear dynamics of the MM and IAZA reaction mechanisms. However, those differences do not affect the applicability of the QSSA to the IAZA reaction mechanism (3). The QSSA can be applied to the nonlinear dynamical system (8a)-(8b) of the IAZA reaction to obtain a single-variable equation for the zymogen depletion:

$$
\frac{\mathrm{d} s}{\mathrm{~d} t} \simeq \frac{\mathrm{d} s^{\varepsilon}}{\mathrm{d} t}=-\frac{k_{2}\left(E_{T}-s^{\varepsilon}\right) s^{\varepsilon}}{K_{M}+s^{\varepsilon}},
$$

which is derived by replacing $c$ in (8a) with its QSS expression, $c^{\varepsilon}$ :

$$
c \simeq c^{\varepsilon}=\frac{\left(E_{T}-s^{\varepsilon}\right) s^{\varepsilon}}{K_{M}+2 s^{\varepsilon}} .
$$

Equation (14) is homologous to the MM equation (1). In the work that follows, we will denote $s^{\varepsilon}$ to be the solution to the zeroth-order approximation, and we will utilize " $s$ " to denote the solution to the mass action model equations. 
We hypothesize that the condition (13) is the validity criterion of the QSSA for the IAZA reaction mechanism (3). The premise for our hypothesis is shown in Figure 5 . We find that $c-s$ phase plane trajectories approach the $c$-nullcline vertically ${ }^{1}$ and then closely follow the invariant manifold governing the steady-state kinetics in both the MM reaction mechanism (2) and IAZA reaction mechanism (3). In both cases, the invariant manifold is practically indistinguishable from the $c$-nullcline, because the reactantstationary approximation holds for the MM reaction mechanism, and the condition (13) holds for the IAZA reaction mechanism. Note that the QSSA approximation for the MM reaction mechanism is valid when the reactant-stationary approximation holds [34]. We explore this hypothesis through scaling analysis [22] in the next section of this paper.
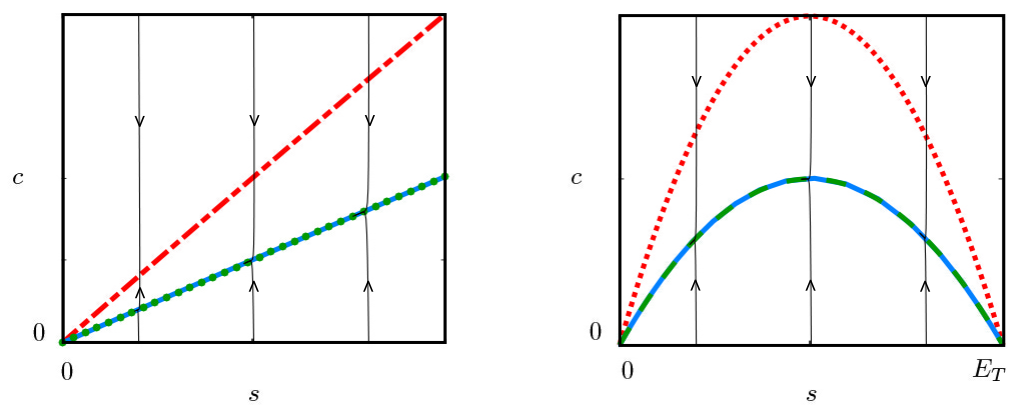

Figure 5: Phase plane trajectories for the MM and IAZA reaction mechanisms when the conditions for the steady-state kinetics are valid. The left panel shows the $c-s$ phase plane for the MM reaction mechanism (2) when the reactant-stationary approximation holds [34]. This approximation guarantees the validity of QSSA. The right panel shows the $c-s$ phase plane for the IAZA reaction mechanism (3) when the condition (13) holds [34]. In both panels, the phase plane trajectories approach and travel through $c$-nullcline which represent the invariant manifold governing the QSS dynamics of the reactions. The initial conditions for both panels are $\left(s_{0}, e_{0}\right)=\{(0.004,0.016),(0.01,0.01),(0.016,0.004)\}$, thus $s_{0}+e_{0}=0.02$ for each pair, implying $E_{T}=0.02$ for the IAZA reaction mechanism. The Michaelis constant for both panels is $K_{M}=2$. Initial conditions and parameters are expressed with arbitrary units for illustrative purposes.

\subsection{Scaling analysis of the governing equations for the autocatalytic zymogen activation}

The QSSA is an important approximation that relies on the natural separation of timescales present in a chemical reaction. For a two-species system, the governing dynamical system consists of two timescales: a fast initial transient, and a slow QSS period [22, 24, 25]. In the case of the IAZA reaction mechanism, like the MM reaction mechanism [22], $s$ remains approximately constant while $c$ builds up rapidly during the initial fast transient. In the QSS phase, there is a measurable change in $s$, and the change in $c$ is dependent on the change in $s$. Formally, we say that $c$ is slaved by $s$ during the QSS phase of the reaction.

We now proceed to scale the nonlinear dynamical system $(8 \mathrm{a})-(8 \mathrm{~b})$ that governs the IAZA reaction mechanism (3). During the initial fast-transient (i.e., when $\left.t \sim t_{f}\right) s$ remains approximately constant to its initial concentration $\left(s \approx s_{0}\right)$. Therefore, we can approximate $\mathrm{d} s / \mathrm{d} t=0$, which reduces $(8 \mathrm{a})-(8 \mathrm{~b})$ to a single differential equation for $c$ :

$$
\frac{\mathrm{d} c}{\mathrm{~d} t} \simeq k_{1}\left[s_{0} e_{0}-\left(K_{M}+2 s_{0}\right) c\right],
$$

with the initial condition $c(0)=0$. The above differential equation can be solved analytically

$$
c(t) \simeq \frac{s_{0} e_{0}}{K_{M}+2 s_{0}}\left[1-\exp \left(-k_{1}\left(K_{M}+2 s_{0}\right) t\right)\right] .
$$

\footnotetext{
${ }^{1}$ Note we have not considered the applicability of a potential QSS reduction with respect to slow product formation in which $k_{2}$ is treated as a small parameter (i.e., in the limit as $k_{2} \rightarrow 0$ ), in which case the nullclines coalesce.
} 
From (16), we estimate the initial fast timescale $t_{f}$ to be

$$
t_{f}=\frac{1}{k_{1}\left(K_{M}+2 s_{0}\right)}
$$

and define the scaled dimensionless time variable for the fast phase of the reaction as:

$$
\tau=\frac{t}{t_{f}}=t k_{1}\left(K_{M}+2 s_{0}\right)
$$

The matching concentrations between the initial fast transient and QSS period are $s\left(t_{f}\right)=s_{0}$ and $c\left(t_{f}\right)=$ $\left(s_{0} e_{0}\right) /\left(K_{M}+2 s_{0}\right)$. These quantities allow us to define the following dimensionless variables:

$$
\bar{s}=\frac{s}{s_{0}} \quad \text { and } \quad \bar{c}=\frac{K_{M}+2 s_{0}}{s_{0} e_{0}} c .
$$

Substituting the scaled dimensionless variables (19) and timescale (18) into (8a)-(8b) yields the scaled nonlinear system governing the IAZA reaction mechanism during the initial fast timescale, $\tau$,

$$
\begin{aligned}
\frac{\mathrm{d} \bar{s}}{\mathrm{~d} \tau} & =\varepsilon \beta(1-\lambda) \bar{s}^{2}+\varepsilon \lambda \beta^{2}(\alpha+2 \sigma \bar{s}) \bar{c}-\varepsilon \beta \bar{s}, \\
\lambda \frac{\mathrm{d} \bar{c}}{\mathrm{~d} \tau} & =\bar{s}-(1-\lambda) \bar{s}^{2}-\lambda \beta(1+2 \sigma \bar{s}) \bar{c} .
\end{aligned}
$$

The dimensionless parameters that appear in (20a)-(20b) are:

$$
\varepsilon \equiv \frac{E_{T}}{K_{M}}, \quad \lambda \equiv \frac{e_{0}}{e_{0}+s_{0}}, \quad \sigma=\frac{s_{0}}{K_{M}}, \quad k=\frac{k_{-1}}{k_{2}}, \quad \alpha \equiv \frac{k}{1+k} \text { and } \beta \equiv \frac{1}{1+2 \sigma} .
$$

Although it appears (at first glance) that $\lambda$ may be a suitable small parameter the validity of the QSSA. However, note that $\lambda=0$ is equivalent to $e_{0}=0$, from which we recover

$$
\bar{s}=0 \quad \text { or } \quad \bar{s}=1 .
$$

Thus, $\lambda=0$ implies that the initial condition lies on the fixed point located at $\left(s_{0}, 0\right)$ whenever $s_{0}$ is nontrivial and $e_{0}=0$. Consequently, $\varepsilon$ arises as a singular perturbation parameter that justifies the applicability of the QSSA via Tikhonov's Theorem. Defining $T=\tau / \varepsilon$ and using the scaled dimensionless variables (19) and parameters (21), we can scale the nonlinear system governing the IAZA reaction mechanism during the slow QSS phase

$$
\begin{aligned}
\frac{\mathrm{d} \bar{s}}{\mathrm{~d} T} & =\beta(1-\lambda) \bar{s}^{2}+\lambda \beta^{2}(\alpha+2 \sigma \bar{s}) \bar{c}-\beta \bar{s}, \\
\varepsilon \lambda \frac{\mathrm{d} \bar{c}}{\mathrm{~d} T} & =\bar{s}-(1-\lambda) \bar{s}^{2}-\lambda \beta(1+2 \sigma \bar{s}) \bar{c} .
\end{aligned}
$$

Note that when $\varepsilon \ll 1, \mathrm{~d} \tilde{c} / \mathrm{d} T$ is $\mathcal{O}\left(\varepsilon^{-1}\right)$ while $\mathrm{d} \tilde{s} / \mathrm{d} T$ is $\mathcal{O}(1)$; these arguments confirm that the small singular parameter $\varepsilon=E_{T} / K_{M} \ll 1$ is a suitable condition for the validity of the QSSA, and delimits the governing nonlinear differential equations (8a)-(8b) for the IAZA reaction mechanism into a fast regime (20a)-(20b), and a slow regime $(23 \mathrm{a})-(23 \mathrm{~b})$.

\subsection{Enzyme assays and the condition for the validity of the QSSA}

In the laboratory, a wide variety of enzyme assays can be used to perform steady-state enzyme kinetics experiments, such as absorbance- and fluorescence-based assays. The equipment and materials for the enzyme assays will vary. Although enzyme assays provide limited information about the fundamental rate 
constants at the microscopic level, they are useful research tools that are commonly used to characterize enzyme or inhibitor activity, or compare the effects of mutations or modifications on enzymatic function.

The most commonly used type of experiment in enzyme kinetics measures the initial rate of an enzymatic reaction as a function of substrate concentration. Initial rate experiments are carried out under experimental conditions where the QSSA is valid. As we mentioned in Section 3, rates are measured after the initial fasttransient by monitoring the substrate depletion or accumulation of product over time. The initial rates measured are plotted versus substrate concentration. The kinetic parameters are determined by fitting the data to the MM equation using nonlinear regression if the mechanism of action follows the MM reaction mechanism (2) [6, 35, for more details $]$

For the IAZA reaction mechanism, the homologous rate law to the MM equation (14) for measuring the substrate depletion is:

$$
\hat{v}_{0}=-\frac{\mathrm{d} s^{\varepsilon}}{\mathrm{d} t}=\frac{k_{2}\left(E_{T}-s^{\varepsilon}\right) s^{\varepsilon}}{K_{M}+2 s^{\varepsilon}} .
$$

To determine the initial rate of autocatalytic zymogen activation using (24), the enzyme assay needs to meet the condition (13) for the validity of the QSSA; this is $s_{0}^{\varepsilon}<E_{T} \ll K_{M}$. This condition requires $K_{M}$ for the IAZA reaction mechanism to be at least one order of magnitude larger than the reactants, $E_{T}$, in the enzyme assay. If no initial estimate for $K_{M}$ is available, a wide range of $E_{T}$ concentrations should be tried and the range refined in subsequent enzyme assays as needed. Alternatively, $E_{T}$ can be more conveniently varied by adding or subtracting zymogen, rather than enzyme, at the start of the reaction.

The curve defined by (24) is shown in Figure 6. It has an unimodal shape that crosses the origin and $E_{T}$, and reaches a limiting rate of $\hat{v}$ at $s_{c}^{\varepsilon *} \simeq E_{T} / 2$ (the same concentration (12) that maximizes $\mathcal{N}_{c}(s)$ from (11)). Thus, when the condition (13) is valid, $s_{c}^{\varepsilon *}=E_{T} / 2, \hat{v}$ yields:

$$
\hat{v} \approx \frac{1}{4} \frac{k_{2} E_{T}^{2}}{K_{M}+E_{T}}
$$

The reaction is approximately zero order in $s_{c}^{\varepsilon *}$ and is said to be saturated, because all of the active sites of the enzyme are occupied by a zymogen.

At very small values of $s^{\varepsilon}$, the denominator of the right-side (24) is dominated by $K_{M}$, so $s^{\varepsilon}$ is negligible compared to both $K_{M}$ and $E_{T}$, and $\hat{v}_{0}$ is directly proportional to $s^{\varepsilon}$ giving an initial slope approximately to

$$
\hat{v}_{0} \approx \frac{k_{2} E_{T}}{K_{M}} s^{\varepsilon} .
$$

This result implies that the reaction is approximately first-order overall at low $s^{\varepsilon}$, and $k_{2} E_{T} / K_{M}$ is a firstorder constant for the reaction. Similar to the MM reaction mechanism, $k_{2} E_{T} / K_{M}$ is the specificity constant of the reaction, and $K_{M} / k_{2} E_{T}$ is the specificity time. This is the time that would be required to consume all of the zymogen if the enzyme were acting under first-order conditions and maintained the same initial rate indefinitely $[1]$.

For the MM reaction mechanism (2), $K_{M}$ is defined operationally as the substrate concentration that corresponds to one half of the limiting rate $v / 2$. For $(24), K_{M}$ does not have the same operational definition. Under QSS conditions, when $\hat{v} / 2$ in $(24), s_{\hat{v} / 2}^{\varepsilon}$ has two values:

$$
s_{\hat{v} / 2}^{\varepsilon-} \simeq \frac{E_{T}}{2}\left(1-\frac{\sqrt{2}}{2}\right) \text { and } s_{\hat{v} / 2}^{\varepsilon+} \simeq \frac{E_{T}}{2}\left(1+\frac{\sqrt{2}}{2}\right) .
$$

The concentrations above are valid under (13), thus under the approximation $s^{\varepsilon *} \simeq E_{T} / 2$ from (12).

\section{Estimation of the reaction completion time for the zymogen activation}

The reaction completion time, $t_{d}$, is a timescale with practical applications in enzyme assays. It can be used to experimentally determine the molar enthalpy $\Delta H_{\text {app }}$ of an enzyme catalyzed reaction in calorimetry assays. An enzyme rate can be determined by measuring the rate of heat generated upon substrate conversion into 


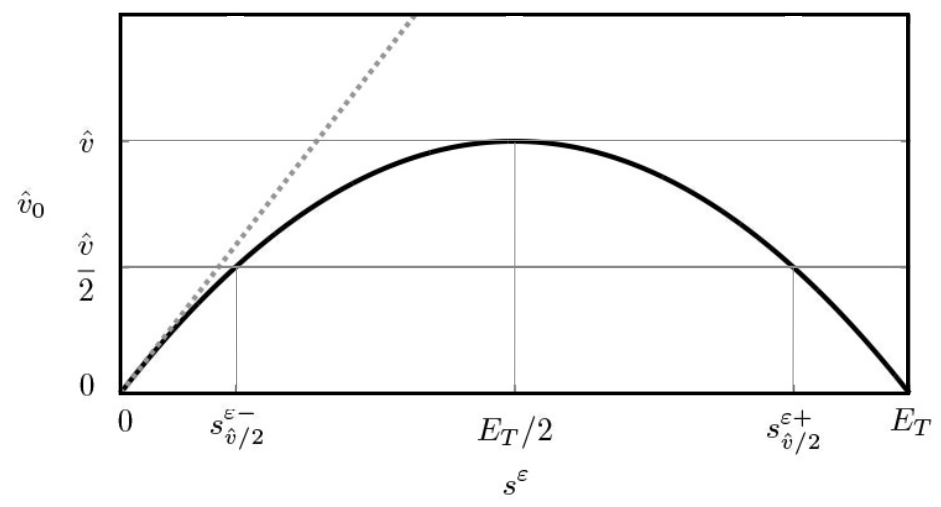

Figure 6: The initial velocity of the IAZA mechanism under QSSA. The initial velocity (24) is represented by the solid black line. The figure shows the limiting rate $\hat{v}$ defined by equation (25) and the operational concentrations $s_{\hat{v} / 2}^{\varepsilon}$ defined as the values $s^{\varepsilon}$ when $\hat{v} / 2(27)$. The initial slope $\hat{v}_{0}$ for $s^{\varepsilon} \approx 0$, defined by equation (26), is marked by the stippled grey line. Kinetic parameters and initial conditions are $k_{2}=1, E_{T}=0.02$ and $K_{M}=2$ with arbitrary units.

product, and estimate $K_{M}$ and $k_{\text {cat }}$ values. The magnitude of $\Delta H_{\text {app }}$ is estimated by allowing the reaction to proceed to completion and then integrating the calorimetry rate signal to obtain the total heat transferred. $\Delta H_{\text {app }}$ is proportional to $t_{d}[36]$.

For the IAZA reaction mechanism (3), the reaction reaches completion when the zymogen is depleted. Therefore, we formally represent, $t_{d}$, as:

$$
t_{d} \approx \frac{\Delta s}{\max \left|\mathrm{d} s^{\varepsilon} / \mathrm{d} t\right|}=\frac{\text { Total change in } s \text { after transient }}{\text { Maximum depletion rate of } s \text { after transient }} .
$$

Defining $\max \left|\mathrm{ds}^{\varepsilon} / \mathrm{dt}\right|$ in (28) to be the limiting rate of the zeroth-order approximation (14), we obtain:

$$
\max \left|\frac{\mathrm{d} s^{\varepsilon}}{\mathrm{d} t}\right|=\left\{\begin{array}{ll}
\frac{k_{2} E_{T}^{2}}{4\left(K_{M}+E_{T}\right)} & \text { for } e_{0} \leq s_{0} \\
\frac{k_{2} e_{0} s_{0}}{K_{M}+2 s_{0}} & \text { for } s_{0}<e_{0}
\end{array} .\right.
$$

The dependency of $\left|\mathrm{d} s^{\varepsilon} / \mathrm{d} t\right|$ on the relation between $s_{0}$ and $e_{0}$ is critical - this is illustrated on FIGURE 7 . The total change in $s, \Delta s$, is equal to the initial zymogen concentration $s_{0}$. Substituting $\Delta s=s_{0}$ and (29) into (28) yields an estimate for the completion time of the zymogen action

$$
\begin{array}{ll}
t_{d}=\frac{4 s_{0}\left(K_{M}+E_{T}\right)}{k_{2} E_{T}^{2}} & \text { for } e_{0} \leq s_{0} \\
t_{d}=\frac{K_{M}+2 s_{0}}{k_{2} e_{0}} & \text { for } s_{0} \leq e_{0}
\end{array}
$$

Thus, the shape of the $c$-nullcline provides two choices for the depletion timescale. It is clear that both (30a) and $(30 \mathrm{~b})$ are in exact agreement when $e_{0}=s_{0}$. Further note that (30b) is homologous to the depletion timescale of the MM reaction mechanism (2) analyzed by Segel [27]. However, if $s_{0}<e_{0}$, then (30a) may be too short to serve as a reasonable depletion timescale (see left panel of Figure 8). In contrast, if $e_{0}<s_{0}$, then $(30 \mathrm{~b})$ provides a more conservative estimate of the depletion timescale in comparison to the more liberal estimate given by (30a) (see right panel of Figure 8). In either case, it is straightforward to show that $t_{f} / t_{d} \rightarrow 0$ as $\varepsilon \equiv E_{T} / K_{M} \rightarrow 0$. Thus, timescale separation is a consistent necessary (but not sufficient) condition for the validity of (14). 


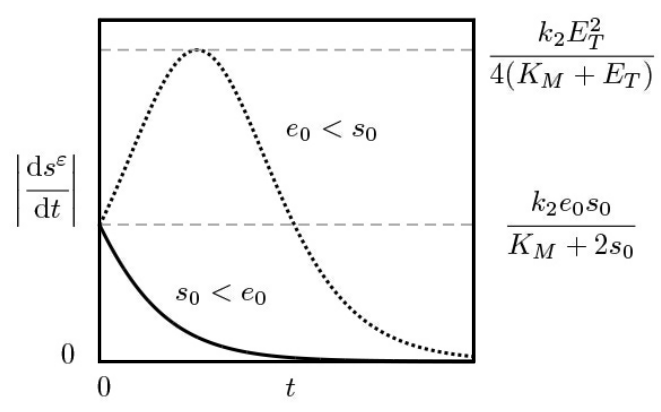

Figure 7: Limiting rate of (14) changes with the relation between $s_{0}$ and $e_{0}$. The figure shows the absolute velocity $\left|\mathrm{d} s^{\varepsilon} / \mathrm{d} t\right|$ for $s_{0}=0.00125<e_{0}=0.00875$ (solid black) and for $e_{0}=0.00125<s_{0}=0.00875$ (dotted black) and the corresponding maxima as defined in (29). Since for both cases $E_{T}=0.01$ the initial velocities are equal, but it equals the maximal absolute velocity only for $s_{0}<e_{0}$; when $e_{0}>s_{0}$ the maximal velocity is achieved at $s \approx E_{T} / 2$. In each situation, the QSSA is satisfied: $k_{1}=k_{2}=k_{-1}=2$, thus the (13) is met. Initial conditions and parameter values are in arbitrary units for illustrative purposes.

\section{Discussion}

The main contribution of this work is the analysis of a model for the autocatalytic zymogen activation (IAZA) reaction mechanism (3), and its comparison with the MM reaction mechanism (2). Although the mechanism of enzyme action is the same for both mechanisms, the nonlinear dynamics of both reaction mechanisms reveal distinct differences. We find that the nature of catalysis is a decisive factor in determining the specific dynamics and geometrical picture of the reaction.

One of the most fundamental dynamical differences between the IAZA and MM reaction mechanism is the number and stability of equilibria points. The MM reaction mechanism only exhibits one stable equilibrium, while the IAZA reaction mechanism exhibits an additional saddle equilibrium point. The existence of these two equilibria creates a heteroclinic orbit of unimodal shape between the saddle equilibrium and stable equilibrium points, which is the invariant manifold that influences the long-time dynamics of the IAZA reaction mechanism (3) in the $c-s$ phase-plane.

The critical result of this work is the derivation of an initial rate equation (14) - homologous to the MM equation - using the QSSA. This expression can be used to estimate the enzyme kinetics parameters by measuring the initial rates of the autocatalytic zymogen activation as a function of zymogen concentrations under the condition (13) for the validity of the QSSA. It should be pointed out that our analysis has been focused on the QSSA due to the fact that the QSSA is commonly utilized as a reduced model to determine kinetic parameters from initial rate experiments. However, there are other forms of the "QSSA" that are commonly implemented, such as the total QSSA, which was originally formulated by Laidler [37], or the reverse QSSA [38]. Subsequent analyses have improved our understanding of the conditions that lead to the validity of the total QSSA [39]. However, the tQSSA is typically utilized to estimate parameters from progress curve experiments, and introduces the experimental challenge of measuring the total substrate concentration. Hence, we have chosen not to analyze the conditions for the tQSSA in this work.

In addition, we also derived a mathematical expression to estimate the completion time of the zymogen activation, which can be used to experimentally determine the molar enthalpy $\Delta H_{\text {app }}$ of the autocatalytic zymogen reaction in calorimetry assays. It is possible that more general timescale estimates can be obtained from the analysis of the total QSSA [40]. However, the timescale estimates derived in this work are sufficient for the region of parameter space that is our primary focus.

\section{Acknowledgement}

Justin Eilertsen is supported by the University of Michigan Postdoctoral Pediatric Endocrinology and Diabetes Training Program "Developmental Origins of Metabolic Disorder" (NIH/NIDDK grant: K12 

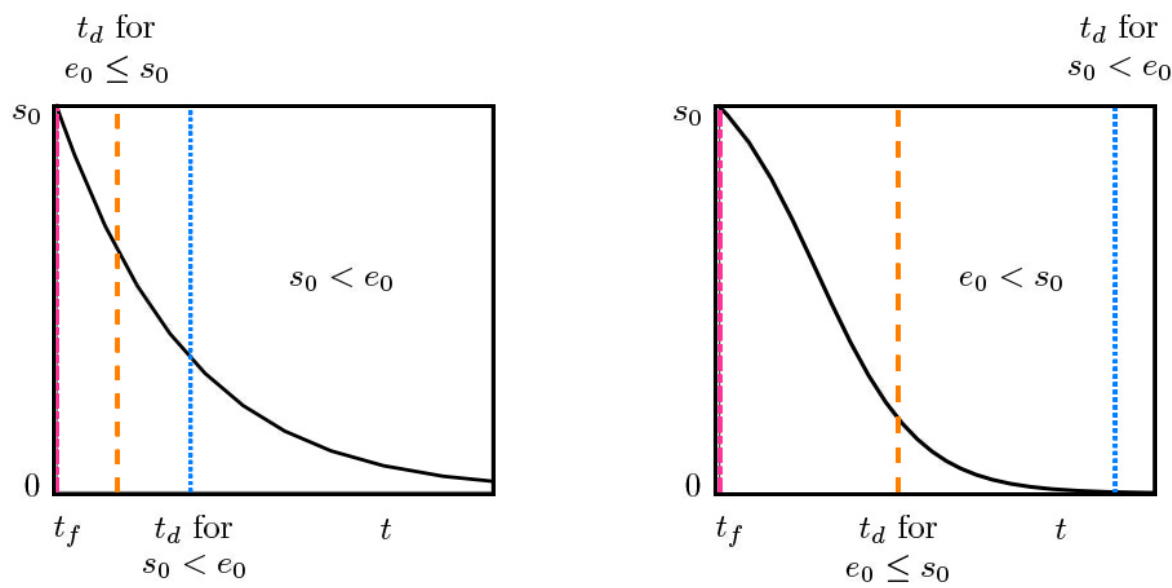

Figure 8: Timescale for completion of the zymogen action as a function initial concentrations. LEFT: Numerically computed progress curve for $s(t)$ (solid black) with the fast $t_{f}$ (dashed pink) timescale marked as vertical lines for reference. Because the initial conditions are $s_{0}=0.0125, e_{0}=0.0875$, the corresponding completion time scale is $t_{d}(30 \mathrm{~b})$ (marked by stippled blue line) must be chosen with respect to the condition $s_{0}<e_{0}$. The dashed orange line shows the complementary estimate for $t_{d}$ from (30a); this time estimate is observed to be too short to characterize a completion of the reaction. The parameters used for the computation are $k_{1}=k_{2}=k_{-1}=1$, thus $K_{M}=2$ and $E_{T}=1$. RIGHT: Same as to the left, but with $s(t)$ computed for $s_{0}=0.0875$, and $e_{0}=0.0125$. The estimate of the $t_{d}$ from (30a) (dashed orange line) approximates the completion time scale more liberally when chosen with respect to the condition $e_{0} \leq s_{0}$, and the complementary estimate from (30b) (dashed orange) provides longer, more conservative estimate.

DK071212). 


\section{References}

[1] A. Cornish-Bowden, Fundamentals of enzyme kinetics, 4th Edition, Wiley-Blackwell, Weinheim, Germany, 2012.

[2] A. Cornish-Bowden, Current IUBMB recommendations on enzyme nomenclature and kinetics, Perspect. Sci. 1 (2014) 74-87.

[3] S. Schnell, Validity of the Michaelis-Menten equation - Steady-state or reactant stationary assumption: that is the question, FEBS J. 281 (2014) 464-472.

[4] W. Stroberg, S. Schnell, On the estimation errors of $K_{M}$ and $v$ from time-course experiments using the Michaelis-Menten equation, Biophys. Chem. 219 (2016) 17-27.

[5] D. L. Purich, Enzyme kinetics: Catalysis and Control, Elsevier, 2010.

[6] H. Bisswanger, Enzyme assays, Perspect. Sci. 1 (2014) 41-55.

[7] A. Cornish-Bowden, One hundred years of Michaelis-Menten kinetics, Perspect. Sci. 4 (2015) 3-9.

[8] J. Al-Janabi, J. A. Hartsuck, J. Tang, Kinetics and mechanism of pepsinogen activation, J. Biol. Chem. 247 (1972) 4628-4632.

[9] C. G. Sanny, J. A. Hartsuck, J. Tang, Conversion of pepsinogen to pepsin. Further evidence for intramolecular and pepsin-catalyzed activation, J. Biol. Chem. 250 (1975) 2635-2639.

[10] B. Kassell, J. Kay, Zymogens of proteolytic enzymes, Science 180 (1973) 1022-1027.

[11] A. R. Khan, M. N. G. James, Molecular mechanisms for the conversion of zymogens to active proteolytic enzymes, Protein Sci. 7 (1998) 815-836.

[12] E. C. Thrower, A. P. E. Diaz de Villalvilla, T. R. Kolodecik, F. S. Gorelick, Zymogen activation in a reconstituted pancreatic acinar cell system, Am. J. Physiol. Gastrointest. Liver Physiol. 290 (2006) G894-G902.

[13] J.-W. Wu, Y. Wu, Z.-X. Wang, Kinetic analysis of a simplified scheme of autocatalytic zymogen activation, Eur. J. Biochem. 268 (2001) 1547-1553.

[14] M. García-Moreno, B. H. Havsteen, R. Varón, H. Rix-Matzen, Evaluation of the kinetic parameters of the activation of trypsinogen by trypsin, Biochim. Biophys. Acta 1080 (1991) 143-147.

[15] M. E. Fuentes, R. Varón, M. García-Moreno, E. Valero, Kinetics of intra-and intermolecular zymogen activation with formation of an enzyme-zymogen complex, FEBS J. 272 (2005) 85-96.

[16] J. Eilertsen, W. Stroberg, S. Schnell, A theory of reactant-stationary kinetics for a mechanism of zymogen activation, Biophys. Chem. 242 (2018) 34-44.

[17] W.-N. Wang, X.-M. Pan, Z.-X. Wang, Kinetic analysis of zymogen autoactivation in the presence of a reversible inhibitor, Eur. J. Biochem. 271 (2004) 4638-4645.

[18] R. Varón, M. A. Minaya-Pacheco, F. García-Molina, E. Arribas, E. Arias, J. Masiá, F. García-Sevilla, Competitive and uncompetitive inhibitors simultaneously acting on an autocatalytic zymogen activation reaction, J. Enzyme Inhib. Med. Chem. 21 (2006) 635-645.

[19] M.-E. Fuentes, R. Varón, M. García-Moreno, E. Valero, Kinetics of autocatalytic zymogen activation measured by a coupled reaction: pepsinogen autoactivation, Biol. Chem. 386 (2005) 689-698.

[20] R. Varón, M. E. Fuentes, M. García-Moreno, F. García-Sevilla, E. Arias, E. Valero, E. Arribas, Contribution of the intra- and intermolecular routes in autocatalytic zymogen activation: Application to pepsinogen activation, Acta Biochim. Pol. 53 (2006) 407-420. 
[21] F. G. Heineken, H. M. Tsuchiya, R. Aris, On the mathematical status of the pseudo-steady hypothesis of biochemical kinetics, Math. Biosci. 1 (1967) 95-113.

[22] L. A. Segel, M. Slemrod, The Quasi-Steady-State Assumption: A case study in perturbation, SIAM Rev. 31 (1989) 446-477.

[23] A. Goeke, C. Schilli, S. Walcher, E. Zerz, Computing quasi-steady state reductions, J. Math. Chem. 50 (2012) 1495-1513.

[24] L. Noethen, S. Walcher, Quasi-steady state and nearly invariant sets, SIAM J. Appl. Math 70 (2009) $1341-1363$.

[25] A. Goeke, S. Walcher, E. Zerz, Classical quasi-steady state reduction - A mathematical characterization, Physica D 345 (2017) 11-26.

[26] A. Goeke, S. Walcher, Quasi-Steady State: Searching for and utilizing small parameters, in: Recent trends in dynamical systems, Vol. 35 of Springer Proc. Math. Stat., Springer, Basel, 2013, pp. 153-178.

[27] L. A. Segel, On the validity of the steady state assumption of enzyme kinetics, Bull. Math. Biol. 50 (1988) 579-593.

[28] A. H. Nguyen, S. J. Fraser, Geometrical picture of reaction in enzyme kinetics, J. Chem. Phys. 91 (1989) $186-193$.

[29] S. Schnell, C. Mendoza, Closed form solution for time-dependent enzyme kinetics, J. Theor. Biol. 187 (1997) 207-212.

[30] A. Dhooge, W. Govaerts, Y. A. Kuznetsov, H. G. E. Meijer, B. Sautois, New features of the software MatCont for bifurcation analysis of dynamical systems, Math. Comput. Model. Dyn. Syst. 14 (2008) $147-175$.

[31] A. Dhooge, W. Govaerts, Y. A. Kuznetsov, W. Mestrom, A. Riet, B. Sautois, MATCONT and CL MATCONT: Continuation toolboxes in MATLAB, Tech. rep., Universiteit Gent - Belgium and Utrecht University - The Netherlands (2006).

[32] M. R. Roussel, S. J. Fraser, Accurate steady-state approximations: Implications for kinetics experiments and mechanism, J. Phys. Chem. 95 (1991) 8762-8770.

[33] S. Schnell, P. K. Maini, A century of enzyme kinetics. Reliability of the $K_{M}$ and $v_{\max }$ estimates, Comments Theor. Biol. 8 (2003) 169-187.

[34] S. M. Hanson, S. Schnell, Reactant stationary approximation in enzyme kinetics, J. Phys. Chem. A 112 (2008) 8654-8658.

[35] J. R. Lorsch, Practical steady-state enzyme kinetics, Methods Enzymol. 536 (2014) 3-15.

[36] M. J. Todd, J. Gomez, Enzyme kinetics determined using calorimetry: A general assay for enzyme activity?, Anal. Biochem. 296 (2001) 179-187.

[37] K. J. Laidler, Theory of the transient phase in kinetics, with special reference to enzyme systems, Can. J. Chem. 33 (1955) 1614-1624.

[38] S. Schnell, P. K. Maini, Enzyme kinetics at high enzyme concentration, Bull. Math. Biol. 62 (2000) 483-499.

[39] A. M. Bersani, E. Bersani, G. Dell'Acqua, M. G. Pedersen, New trends and perspectives in nonlinear intracellular dynamics: one century from Michaelis-Menten paper, Continuum Mech. Therm. 27 (2015) 659-684.

[40] J. A. M. Borghans, R. J. De Boer, L. A. Segel, Extending the quasi-steady state approximation by changing variables, Bull. Math. Biol. 58 (1996) 43-63. 